\section{Bejel, a Nonvenereal Treponematosis, among Men Who Have Sex with Men, Japan}

\author{
Takuya Kawahata, Yoko Kojima, \\ Keiichi Furubayashi, Koh Shinohara, \\ Tsunehiro Shimizu, Jun Komano, \\ Haruyo Mori, Kazushi Motomura
}

Author affiliations: Osaka Institute of Public Health, Osaka, Japan (T. Kawahata, Y. Kojima, H. Mori, K. Motomura); Sonezaki Furubayashi Clinic, Osaka (K. Furubayashi); Kyoto City Hospital, Kyoto, Japan (K. Shinohara, T. Shimizu); Nagoya Medical Center, Nagoya, Japan (J. Komano); Osaka University of Pharmaceutical Sciences, Takatsuki, Japan (J. Komano)

DOI: https://doi.org/10.3201/eid2508.181690

Bejel, an endemic treponematosis caused by infection with Treponema pallidum subspecies endemicum, has not been reported in eastern Asia and the Pacific region. We report local spread of bejel among men who have sex with men in Japan. Spread was complicated by venereal syphilis.

$T$ reponema pallidum subspecies pallidum is the causative agent of venereal syphilis. Globally, syphilis remains a disease of heterosexual persons in low-to-middle-income countries. Epidemics of syphilis among men who have sex with men (MSM) occur in high-income settings (1). Other Treponema species cause nonvenereal endemic treponematosis (also called bejel, nonvenereal syphilis, or endemic syphilis, caused by $T$. pallidum subsp. endemicum), yaws ( $T$. pallidum subsp. pertenue), and pinta (T. carateum). These pathogens are morphologically and serologically indistinguishable (2). Clinically, there is little need to differentiate them. However, it is useful to differentiate them from a public health standpoint because their infection routes vary. For this purpose, a nucleic acid test is useful (3).

Bejel was eradicated in Europe in the 20th century but was prevalent there in the 16th century (4). Bejel is still prevalent in dry and hot areas, such as the Sahel region in western Africa, part of Botswana, Zimbabwe, and the Arabian Peninsula (5). The main route of transmission is direct skin-to-skin contact. Bejel can be transmitted sexually, but this route has not been studied because bejel affects mainly children. Only a few case reports of bejel have been reported in non-endemic areas since 1999, including France (3), Canada (6), and Cuba (7). Bejel in France was attributed to an imported case from Pakistan, and in Canada to an imported case from Senegal, whereas transmission in Cuba was regionalized. No patient with nonvenereal treponematosis has been reported in Japan.
In Japan, syphilis has been reemerging since 2010 (8). However, little attention has been paid to nonvenereal treponematosis. We thus conducted a molecular epidemiologic study to characterize the genotypes of T. pallidum subsp. pallidum among patients with venereal syphilis after 2011 (9).

The study protocol was approved by the Ethical Review Board of Osaka Institute of Public Health. We tested specimens from patients suspected of having or given a diagnosis of syphilis by using nucleic acid amplification tests for $T$. pallidum subsp. pallidum specific for the TpN47 and polA gene regions. We performed molecular genotyping of T. pallidum subsp. pallidum strains based on the nucleic acid sequences of the tp0548 and tp0856 gene regions $(3,10)$.

Phylogenetic analysis showed that, of 58 isolates from nucleic acid test-positive specimens, 5 isolates $(8.6 \%)$ were $T$. pallidum subsp. endemicum and different from $T$. pallidum subsp. pallidum and T. pallidum subsp. pertenue. We concluded that the 5 patients from whom these strains were isolated had bejel (Figure).

All 5 bejel patients were men from Japan 20-40 years of age; all were MSM. One patient was identified in 2014, another 3 in 2017, and 1 patient in 2018. Two of the patients identified in 2017 were in the secondary stage of the disease; the other 3 were in the primary stage. Clinical manifestations of the 3 patients in the primary stage were penile erosion or ulcer. The 2 patients in the secondary stage had systemic rashes and lymphadenopathy, in addition to pubic and perineal symptoms.

For serologic tests at admission, the 3 primary-stage patients showed negative results for the rapid plasma reagin test ( $<1.0$ unit). Of these patients, 2 showed negative results of the T. pallidum latex agglutination test ( $<10$ units) and 1 had a titer of 35.7 units. The 2 secondary-stage patients had positive results for the rapid plasma reagin test, and their $T$. pallidum latex agglutination test values were $2.4 \times 10^{3}$ and $20.8 \times 10^{3}$ units

The first patient lived in Yamaguchi Prefecture. The other 4 patients lived in the Kansai area, including Osaka, Kyoto, and Hyogo Prefectures. Although the residential geographic areas were remote, the suspected locale of treponemal infection was the Kansai area, namely the city centers of Osaka and Kyoto. The 2018 patient was HIV positive. None of the patients had a history of overseas travel for a long period. All 5 isolates had a mutation conferring azithromycin resistance. The 3 patients who were followed up responded well to standard therapy with penicillin.

These data strongly suggest that $T$. pallidum subsp. endemicum is transmitted domestically in Japan by MSM. Our findings provide molecular epidemiologic evidence for a local spread of T. pallidum subsp. endemicum in eastern Asia and the Pacific region.

Clinical manifestations of venereal syphilis and bejel are similar, especially in the early stage for adults, which 


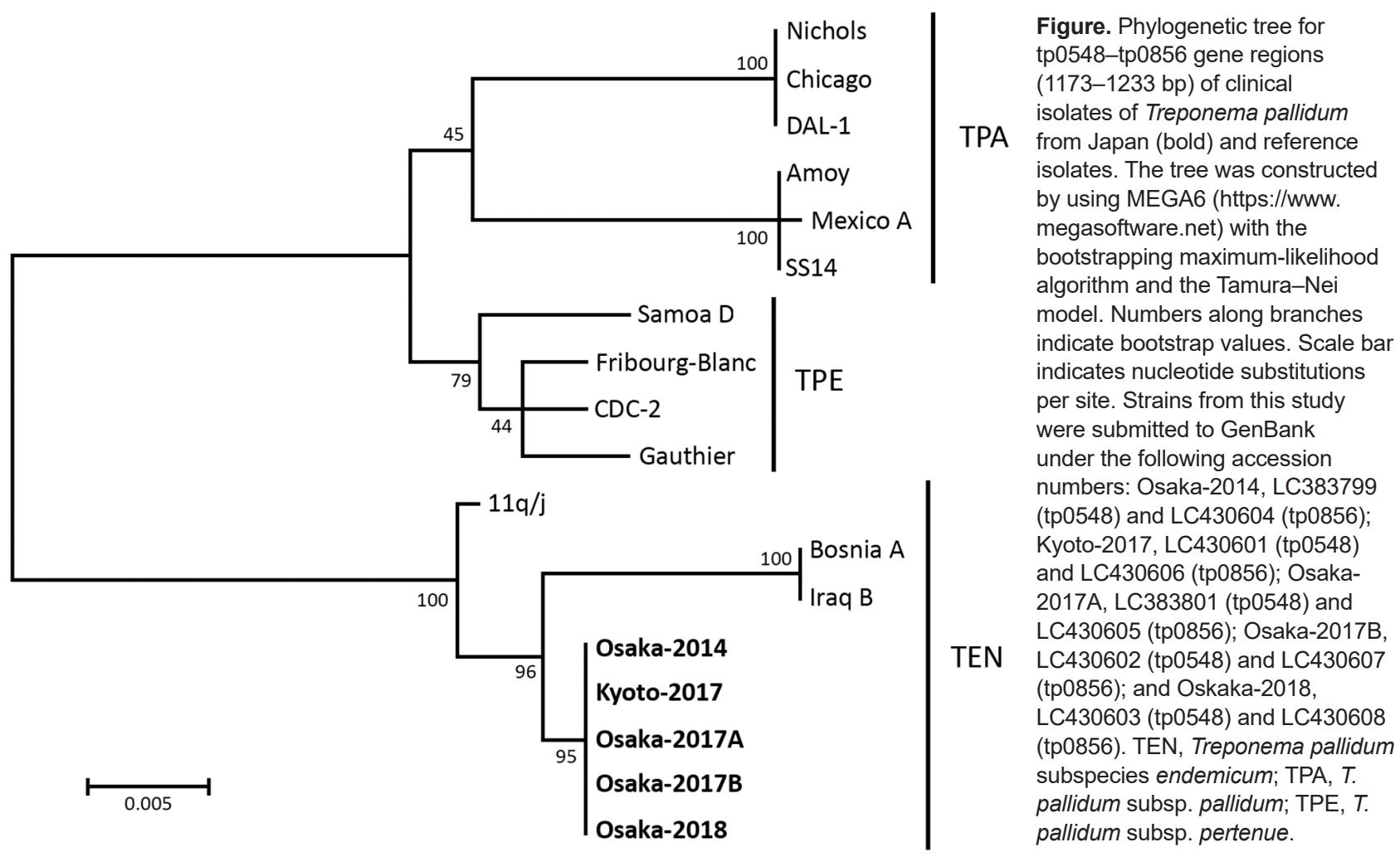

makes diagnosis difficult (7). Infectious diseases that have been historically not considered to be sexually transmitted infections (STIs), such as amebiasis, hepatitis A, and shigellosis, often show manifestations of STIs. Likewise, bejel might be changing from an endemic tropical disease to a global STI.

Treatment for venereal syphilis is also effective for bejel. For the 5 patients we report, laboratory test results showed a strong correspondence to the stage of bejel disease progression. Clinicians should be aware of the spread of nonvenereal treponematosis worldwide, especially in low-prevalence areas. Nucleic acid tests that can differentiate $T$. pallidum strains might be helpful $(3,10)$. Molecular epidemiology might help determine which populations are affected and provide an effective means to prevent the further spread of treponematosis.

This study was partially supported by grants-in-aid for research on HIV/AIDS from the Ministry of Health, Labour and Welfare of Japan (grants H26-AIDS-006 and H29-AIDS-007) to T.K. and a grant from the Daido Life Welfare Foundation for Regional Health and Welfare Research in 2017 to Y.K.

\section{About the Author}

Dr. Kawahata is a principal research scientist at the Osaka Institute of Public Health, Osaka, Japan. His primary research interests are HIV infections, sexually transmitted infections, and syphilis.

\section{References}

1. Fenton KA, Breban R, Vardavas R, Okano JT, Martin T, Aral S, et al. Infectious syphilis in high-income settings in the 21 st century. Lancet Infect Dis. 2008;8:244-53. https://doi.org/10.1016/ S1473-3099(08)70065-3

2. Mitjà $\mathrm{O}$, Šmajs D, Bassat Q. Advances in the diagnosis of endemic treponematoses: yaws, bejel, and pinta. PLoS Negl Trop Dis. 2013;7:e2283. https://doi.org/10.1371/ journal.pntd.0002283

3. Mikalová L, Strouhal M, Oppelt J, Grange PA, Janier M, Benhaddou N, et al. Human Treponema pallidum $11 \mathrm{q} / \mathrm{j}$ isolate belongs to subsp. endemicum but contains two loci with a sequence in TP0548 and TP0488 similar to subsp. pertenue and subsp. pallidum, respectively. PLoS Negl Trop Dis. 2017;11:e0005434. https://doi.org/10.1371/journal.pntd.0005434

4. Lipozenčić J, Marinović B, Gruber F. Endemic syphilis in Europe. Clin Dermatol. 2014;32:219-26. https://doi.org/10.1016/ j.clindermatol.2013.08.006

5. Giacani L, Lukehart SA. The endemic treponematoses. Clin Microbiol Rev. 2014;27:89-115. https://doi.org/10.1128/ CMR.00070-13

6. Fanella S, Kadkhoda K, Shuel M, Tsang R. Local transmission of imported endemic syphilis, Canada, 2011. Emerg Infect Dis. 2012;18:1002-4. https://doi.org/10.3201/eid1806.111421

7. Noda AA, Grillová L, Lienhard R, Blanco O, Rodríguez I, Šmajs D. Bejel in Cuba: molecular identification of Treponema pallidum subsp. endemicum in patients diagnosed with venereal syphilis. Clin Microbiol Infect. 2018;24:1210.e1-5. https://doi.org/10.1016/j.cmi.2018.02.006

8. Takahashi T, Arima Y, Yamagishi T, Nishiki S, Kanai M, Ishikane $\mathrm{M}$, et al. Rapid increase in reports of syphilis associated with men who have sex with women and women who have sex with men, Japan, 2012 to 2016. Sex Transm Dis. 2018;45:139-43. https://doi.org/10.1097/OLQ.0000000000000768 
9. Kojima Y, Furubayashi K, Kawahata T, Mori H, Komano J. Circulation of distinct Treponema pallidum strains in individuals with heterosexual orientation and men who have sex with men. J Clin Microbiol. 2019;57:e01148-18. https://doi.org/10.1128/ JCM.01148-18

10. Staudová B, Strouhal M, Zobaníková M, Cejková D, Fulton LL, Chen L, et al. Whole genome sequence of the Treponema pallidum subsp. endemicum strain Bosnia A: the genome is related to yaws treponemes but contains few loci similar to syphilis treponemes. PLoS Negl Trop Dis. 2014;8:e3261. https://doi.org/10.1371/ journal.pntd.0003261

Address for correspondence: Takuya Kawahata, Osaka Institute of Public Health, 1-3-69 Nakamichi, Higashinari-ku, Osaka 537-0025, Japan; email:kawahata@iph.osaka.jp

\section{Multidrug-Resistant Klebsiella pneumoniae ST307 in Traveler Returning from Puerto Rico to Dominican Republic}

\section{Rita Rojas, Nenad Macesic, Gilda Tolari, Anel Guzman, Anne-Catrin Uhlemann}

\author{
Author affiliations: Hospital General Plaza de la Salud, Santo \\ Domingo, Dominican Republic (R. Rojas, G. Tolari, A. Guzman); \\ Monash University, Melbourne, Victoria, Australia (N. Macesic); \\ Columbia University Medical Center, New York, New York, USA \\ (N. Macesic, A.-C. Uhlemann)
}

DOI: https://doi.org/10.3201/eid2508.171730

We report $b / a_{\mathrm{KPC}-2}$-harboring carbapenem-resistant Klebsiella pneumoniae in an emerging sequence type 307 lineage in a traveler returning from Puerto Rico to the Dominican Republic. Phylogenetic analyses indicate regional dissemination of this highly drug-resistant clone across the Americas, underscoring the need for adequate surveillance and infection control efforts to prevent further spread.

$\mathrm{C}$ arbapenemase-resistant Enterobacteriaceae (CRE), in particular carbapenem-resistant Klebsiella pneumoniae (CRKp), represent a serious threat to public health (1). CRKp infections have been associated with high mortality rates, up to $50 \%$ in some studies (2). In resource- limited regions, such as the Dominican Republic, multiple challenges hinder efforts to contain CRE infections, including lack of novel antimicrobial drugs, inability to monitor drug levels of potentially toxic treatment regimens, and absence of molecular tools to investigate outbreaks and potential spread.

In fall 2015, a 66-year-old woman with diabetes mellitus, hepatitis $\mathrm{C}$ virus infection, and end-stage renal disease on hemodialysis was admitted to a hospital in the Dominican Republic for fever, anorexia, chills, and myalgia. On day 3, her blood culture tested positive for K. pneumoniae. She had been admitted to a hospital in Puerto Rico a few months before and had been treated for a multidrug-resistant bacterial infection.

The $K$. pneumoniae isolate from the patient was nonsusceptible to all tested antimicrobial drugs except polymyxins (Appendix Table 1, http://wwwnc.cdc.gov/EID/ article/25/8/17-1730-App1.pdf). We began combination therapy with a loading dose of colistin, then $100 \mathrm{mg}$ postdialysis, plus ertapenem (150 $\mathrm{mg}$ postdialysis) and fosfomycin ( $2 \mathrm{~g} 3 \times / \mathrm{d})$. We implemented infection control measures by placing the patient in a single room and using gloves, gowns, masks, and a dedicated stethoscope. Despite initial improvement, the patient died on day 25 after admission.

Whole-genome sequencing revealed that the patient isolate, NR6025, was of the emerging sequence type 307 (ST307) (3) and closely related ( $\leq 185$ SNPs) to several international ST307 isolates of similar phenotype (Figure). Of note, this isolate was most closely related, within 36 SNPs, to an isolate recovered from a patient in New York, NY, USA, who also had been hospitalized in Puerto Rico in 2016 (4). This finding raises the possibility that both patients acquired CRE in Puerto Rico and their infections subsequently developed in their home countries.

In silico resistance gene detection demonstrated that $b l a_{\mathrm{KPC}-2}$, on Tn4401e, was likely the mechanism of carbapenem resistance for this isolate. Moreover, the meropenem MIC was $>32 \mu \mathrm{g} / \mathrm{mL}$, consistent with high carbapenem MICs observed in the ST307 Tn4401e isolates (4) from New York, suggesting association with a strong promoter. In addition, the isolate harbored a large repertoire of acquired-resistance genes, including additional $\beta$-lactamase genes CTX-M-15, SHV-100, OXA-1, and TEM-1D (Appendix Table 1). The isolate contained IncFIBK, ColRNA1, and IncA/C2 plasmid replicons; IncA/C plasmid encodes for $b l a_{\mathrm{KPC}-2}, b l a_{\mathrm{TEM}}, s u l I, a a d B, a a c 6$, and qacE, which has been implicated in chlorhexidine resistance.

A case of CRKp was described from Medellin, Colombia, in 2005, and subsequent CRKp infections have been reported in Mexico, in South America in Brazil, Argentina, and Venezuela, and in the Caribbean in Cuba, Puerto Rico, and Trinidad and Tobago (5-7). In many of these studies, CRKp isolates were mainly accounted for 